\title{
Learning by Feeling: Evoking Empathy with Synthetic Characters
}

\author{
Ana Paiva, João Dias, Daniel Sobral \\ Instituto Superior Técnico and INESC-ID \\ Av. Prof. Cavaco Silva, IST, Taguspark \\ Porto Salvo, Portugal \\ ana.paiva@inesc-id.pt \\ Ruth Aylett \\ Center for Virtual Environments, \\ University of Salford, UK \\ Sarah Woods \\ Adaptive Systems Research Group, \\ University of Hertfordshire, UK \\ S.N.Woods@herts.ac.uk \\ Lynne Hall \\ School of Computing and Technology, \\ University of Sunderland, Sunderland, UK \\ lynne.hall@sunderland.ac.uk \\ Carsten Zoll \\ Institute of Theoretical Psychology, \\ University of Bamberg, Germany \\ carsten.zoll@ppp.uni-bamberg.de
}

\begin{abstract}
Virtual environments are now becoming a promising new technology to be used in the development of interactive learning environments for children. Perhaps triggered by the success of computer games, VEs are now seen as an emergent and engaging new way by which children learn experimental sciences and other disciplines. Inhabiting these IVEs there can be agents or intelligent characters, that are responsible for events that happen in the environment and make it not predictive or completely controlled. However, to build such environments, in particular if populated by synthetic characters, one needs to carefully address the problem of how do the learners respond to the characters in the virtual environment. Do learners like the characters? Do learners identify themselves with characters in virtual en-
\end{abstract}


vironments? This relation between learners and characters in virtual environments can be studied in several perspectives. In this paper, we will focus primarily on the issue of empathy as one desirable aspect of the affective interaction between learners and synthetic characters. In particular we will defend that in order for such affective relations to happen, characters should be created and designed taking into account what we call the proximity factor. This is based on the fact that children are found to respond more empathically to those that are perceived as similar to the self than those who are perceived as dissimilar [2]. This appears to be the case when similarity is defined in terms of a shared characteristic, such as sex [6], race or in terms of shared personal experiences [2]. Thus, designing characters aiming at pedagogical empathic interactions, we should careful address how close the learner will feel with the synthetic characters developed both in terms of situation, behaviour or even physical appearance.

In order to illustrate this factor in eliciting emotional reactions to synthetic characters, we will present a specific system called FearNot!. FearNot! was developed to address the difficult and often devastating problem of bullying in schools. By using role playing and synthetic characters in a 3D environment, FearNot! allows children from 8 to 12 to experience a virtual scenario where they can witness (in a third-person perspective) bullying situations. To build empathy into FearNot! we have considered the following components: agent's architecture; the characters' embodiment; the environment itself and emotionally charged situations. All these elements were build to allow for a stronger proximity with the user and the system. In this paper we will focus primarily on this problem and report some results achieved in the evaluation done with 127 children and 95 adults on the system.

\section{Introduction}

Intelligent Virtual Environments (IVEs) bring new challenges to the way we use technology in educational contexts, promoting and creating new learning experiences where experimentation and presence are explored. One of the big advantages of IVEs is that they offer a safe place where learners can explore and understand through experimentation without the dangers or problems of the real situations. Moreover, when IVEs are augmented with contextual information, questions and activities, they can engage learners in entertaining and motivating experiences, otherwise often considered as boring and uninteresting. Like computer games games, IVEs may allow learners to get immersed and interact in synthetic worlds using a set of interaction facilities, such as move, talk and specific actions with other characters. Inhabiting these IVEs there can be agents or intelligent characters, that are responsible for events that happen in the environment and make it not predictive or completely controlled. Characters can be given the roles of teacher; helpers, companions, elements in the simulated worlds, or even friends. They become the part of the environment giving liveness in the interaction with the learners.

Given these aspects of Intelligent Virtual Environments they have been used successfully in science and technology education (see for example [23] [15] [12]), as a boost to these often scarcely appreciated areas of knowledge. However, when populated with animated characters IVEs may also offer users a safe environment where 
they can explore and learn through experiential and entertaining activities in areas such as social learning. However, this can only be achieved if the learners feel that the environments do meet their expectations in terms of real to life and the synthetic characters are believable enough to display the appropriate and expected behaviours. Thus, when considering Social learning using IVEs, believability is perhaps one of the main goals to attain. A believable character has been defined as a character that gives the illusion of life and allows the user's suspension of disbelief [3]. This quest for believability has indeed been the Holy Grail of the area of synthetic characters for years. However, given the nature of the concept (believability), several aspects are at stake. One of them is the character's appearance. Are more realistic characters more believable? And about cartoon like characters?. One second factor that leads to belivability is the character's autonomy. Again, some results show that the more autonomous may seem more believable. See for example the case of the tamagochis. However, autonomy is difficult to achieve in synthetic characters as there are tremendous technological difficulties, such as for example the speech generation. Often, completely scripted characters lead to more realistic and believable situations.

One other aspect to consider for believability is the character's perceivable actions and expressions. Expressivity is perhaps one of the most challenging problems of synthetic characters, and work such as [21] [4] or [7] go in that direction. In particular, the expression of emotions is understood as fundamental to achieve some degree of believavility. In fact, according to Thomas and Johnston [27], animators from Disney, there are three important points when expressing emotions: (1) the emotional state of the character must be clearly defined, in such a way that is undoubtedly perceived by the viewer; (2) the emotional state affects the reasoning process and consequences must be perceivably reflected in the actions of the characters; and (3) emotions can be accentuated or exaggerated, to clearly communicate to the viewer the emotional state of the character. Another element is personality. A coherent character, that acts according to its personality will be more believable [19] than a character that has no long term coherence in its behaviour.

But on a whole, it is not so much one property or another that matters, but rather the combination of all these factors, that together provide ingredients for building believability in a Pedagogical Virtual environment with synthetic characters.

However, when we watch a film, or read a book, we do not only suspend our disbelief and look at the characters as "alive", but we also establish emotional relations with the characters, even if they are ducks, ants, cartoon or realistic. We feel sad when they are sad, angry when something unfair is done to our favourite character, disappointed when our character didn't achieve what we wanted, and so on. That is, we put ourselves in the shoes of the characters, and feel emotions about what is happening to them. So, together with emotional expression, autonomy and personality, we believe that "empathy" is also an important factor that can lead characters to become believable.

Empathy can be defined in broad terms as "An observer reacting emotionally because he perceives that another is experiencing or about to experience an emotion". Another, less broad, definition is given by Wispé that described empathy as "the process whereby one person 'feels her/himself into the consciousness of another person" [29].

Although animators and film makers have been doing it for years, creating embod- 
ied lifelike autonomous characters that have the power to make the user feel emotional reactions is still an unexplored research challenge, in particular, for educational purposes. There are several factors to take into account and each one of them is, per se, a research topic. In this paper we will discuss the role of empathy in the construction of synthetic characters in VEs for educational purposes, focusing primarily on how to build characters to evoke emotional responses (empathic responses) .

So, our main problem is:

How can we build synthetic characters that are able to evoke and establish empathic relations with learners in a virtual environment?

To illustrate our approach to this problem we will rely on one particular example of a pedagogical system, FearNot!, developed for addressing bullying problems in schools using an interactive virtual storytelling environment. We will describe some of the issues surrounding the development of FearNot! and the approach taken in terms of character's development. By focusing on the proximity factor we also describe the results achieved by the evaluation of one first prototype of the system. The results show that children do relate more strongly with the characters than adults, and consider the characters, the situations and the environment believable. The results also show that children do exhibit more empathy towards the developed characters than adults.

This paper is organised as follows: first we will review some related work, which we considered relevant for our problem. Secondly we will describe the application FearNot! in order to illustrate the problem, the situations and contextualize the research presented. Then, we will describe our approach to building synthetic agents that were designed to evoke empathy, not only because of their behaviour and architecture, but also because of their physical appearance and the situations chosen. Finally, we will describe an evaluation performed with a small prototype of FearNot! that shows the relation children had with the characters and draw some directions for future work.

\section{Related work}

One of the most promising arenas for exploring believability of synthetic character is education. The characters, when immersed into a learning environment can react to the learner's performance, respond adequately to the learner's actions, help and advice the learner in a more human like manner.

The benefits of using a character, by contrast to plain learning applications was well explored by Lester who studied the effect of the presence of a lifelike character in a interactive learning environment. Using an agent called Herman the Bug [14], who inhabited a botanical world, through learning session, Herman would provide guidance and advice to the learner in a quite lively and expressive manner. Herman used a sequencing engine to dynamically sequence the animated set of behaviours and thus give the illusion of life.

But perhaps the most influential pieces of work in the area of synthetic characters in education was the work by J. Rickel [12] with the agent Steve. Steve (Soar Training Expert for Virtual Environments) is a pedagogical agent that helps students to perform 
physical, procedural tasks in a operations room where he had to operate and repair complex equipment. Learners, whilst interacting with Steve were immersed in a 3D computer simulated world where their work environment was modelled and learners were able to practice their stills with the system. In spite of its relevance, this work did not address carefully the relation established between students and Steve, and the facial expressions of Steve were somehow quite limited.

Another very interesting example of the presence of children in a VE is the project NICE, (The Narrative Immersive Constructionist/Collaborative Environments) that was an exploratory learning environment for children. In such environment, the children are asked to collaboratively construct, cultivate and maintain a healthy virtual garden [23]. The NICE project combined the ideas of constructionism, narrative, and collaboration within a single environment. The interactions with the system take place in a CAVE, a multi-person room-size virtual reality system consisting of three walls and a floor. The users wear special lightweight stereo-glasses, which allow them to see the virtual and real world without any transition and carry a special hand-held device for interaction called the wand. The children are represented in the virtual world by an avatar, which interact with the many intelligent guides - who help them to maintain the ecosystem.

Although most of the learning environments with synthetic characters have been developed in domain areas of science and technology, the role of these characters can be also explored in social learning setting. One example for this is the well known Carmen's Bright IDEAS, an interactive health intervention designed to improve the problem solving skills of mothers of pediatric cancer patients (Marsella, 2000). The Bright IDEAS method is a method of social decision-making and problem solving, applied in clinical settings, via a series of one-on-one sessions with trained counsellors. To provide mothers with a way to explore decision making using the Bright Ideas Method, an interactive narrative was produced. A professional scriptwriter aided by the clinical professionals, who conceived and administered the Bright IDEAS method, developed the story underneath the Carmen's Bright IDEAS. The story is organized into three acts: - in the first act, the learner (a mother) is presented with a sequence of situation vignettes, which show some back-story for the Carmen character. This sequence aims at helping the learner to identify and empathise with Carmen. The second act takes place in the counsellor's office, where Carmen discusses her problems with Gina, the counsellor. This discussion is done by first selecting a problem to analyse, and develops through the evaluation of the possible solutions. The interactivity of this application varies from act to act, but in general the user is always able to influence the flow of action within the story and to develop a model of Carmen's emotional state that will guide the interactions during the story progression. The story progression in this phase is highly dependent on Gina's judgment since she is the one that based on the thoughts selected, decides if a further elaboration is required or if the current topic of the Bright IDEAS method is already sufficiently discussed. The application was tested in a real setting and the results showed that the mothers identified themselves with Carmen and with Carmen's problems and found her solutions believable. 


\section{FearNot!}

In order to illustrate our approach to the development of empathic agents in pedagogical VEs, in particular, the proximity factor, we will present an application, FearNot!, where empathy is at the center of the interaction between learners and characters.

FearNot! is a computer application being developed to tackle and eventually help to reduce bullying problems in schools. Bullying behaviour is characterised as "a repeated action that occurs regularly over time, and usually involves an imbalance in strength, either real or perceived" [8]. Bullying has associated with it a wide variety of behaviours such as hitting, kicking or punching, in the case of direct bullying, or, in relational bullying, social exclusion or malicious rumor spreading. It is this a repetition of violent pressure over time (physical or verbal) that distinguishes bullying from other types of violence, making its consequences so potentially dangerous and enduring.

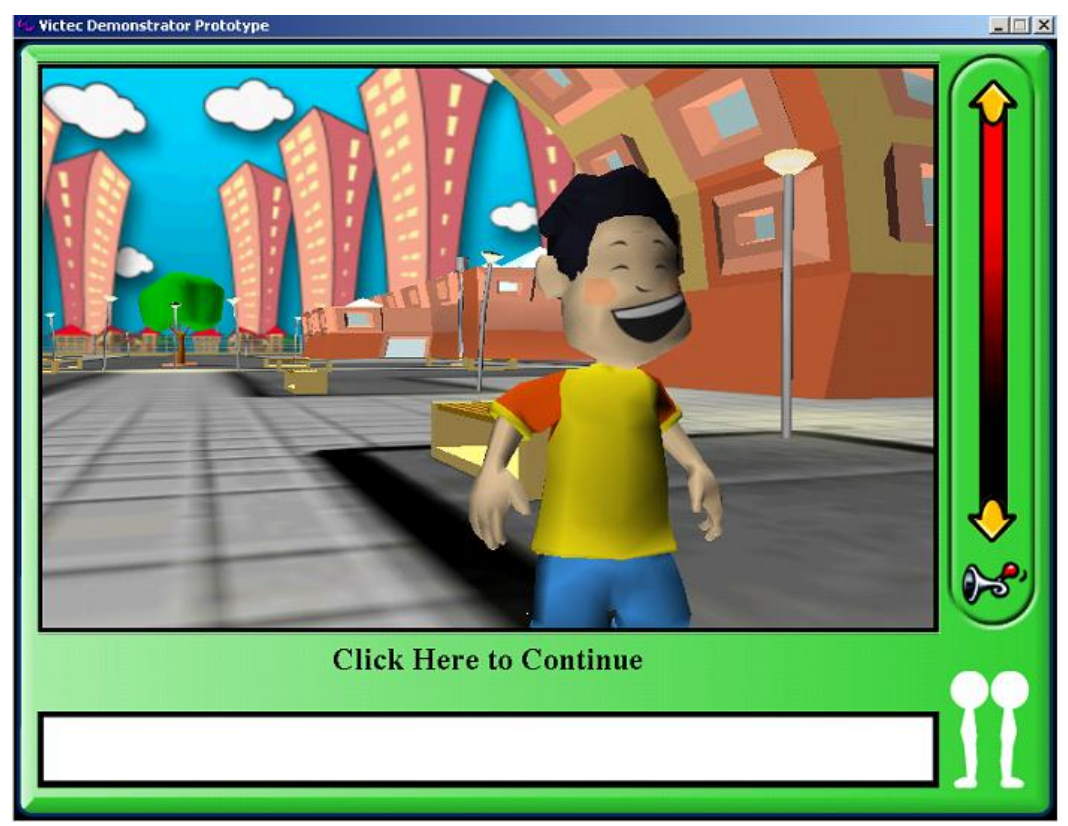

Figure 1: A first prototype of the fearNot demonstrator

A wide range of anti-bullying initiatives have been developed, focusing on the victim, the bully or the whole problem. One approach uses live performance to dramatize the problem, with actors presenting a narrative, followed by workshops in which groups discuss the story. In an extension of this, Boal's Forum Theatre [5] allows each group to take responsibility for one of the characters and to meet with the actors 'in role' between episodes of the story. Such an approach is however expensive and hard to organise, while the presence of the group is often intimidatory (some members may be bullying others) and can even emphasize existent conflicts.

Given the extension of the problem, a EU funded project (VICTEC) was created to 
add to the already existing initiatives, a new one, based on Intelligent Virtual Environments. Through the implementation of a virtual Forum Theatre for education, one can hope to create a safe environment in which individual children can explore different perspectives on bullying behaviour. These were the main foundations that forged the VICTEC project. Using state-of-the-art 3D interactive graphics and synthetic actors (see Figure 3), we expect to achieve individual interaction based on creating empathy with the characters.

Thus, the overall pragmatic objective of the development of FearNot!, was to build an anti-bullying demonstrator in which children age 8-12 experience a virtual scenario where they can witness (from a third-person perspective) bullying situations. To avoid group pressure and enable individualized interaction, the experience is for a single user. The child acts as an invisible friend to a victimized character, discussing the problems that arise and proposing coping strategies. Note that in bullying situations there are quite clear identifiable roles: the bully, the victim, bully-victim (a child that is sometimes the victim and sometimes the bully) and bystander.

The scenario begins by introducing the child to the school environment and the characters, providing a starting context (see Figure 3). This initial presentation provides the background needed about the characters in the story (a description of who is the bully, the victim, and so on). Then, the episodes start. The whole session is developed one episode after another. Within an episode, the child is mostly a spectator of the unfolding events (the narrative emerges from the actions of the participant characters). After each episode, however, the victim will seek refuge in a resource room (identified as a library) where a personalized conversation with the user can occur.

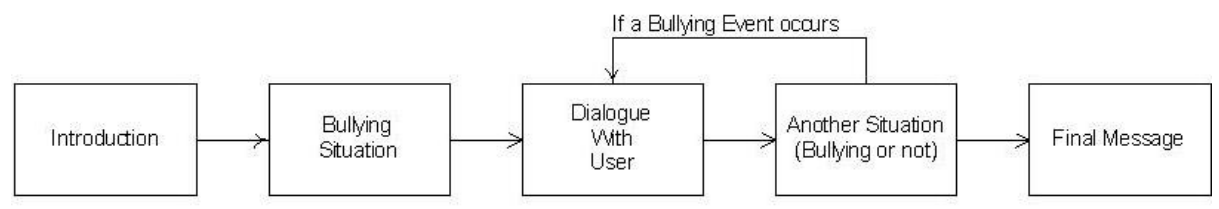

Figure 2: Interacting with FearNot!

Then, the child takes the role of a friend of the victim advising her on what to do. A short dialogue takes place between the two, where the victim raises the main events that occurred in the previous episode and asks for the child's (learner) opinion and suggestions for future behaviour. The dialogue established between the child user and the victim character is done based on a set of patterns of response to bullying situations, however, allowing the children to express the reasons and the expectations for the advice given to the character victim. Nevertheless, note that the victim is clearly recognized as a believable self, with its own personality and behaviour, and thus may decide to reject the child's suggestions (see Figure 2.

Each dialogue finishes with a decision that influences the character's behaviour in future episodes. Thus, episodes are not pre-scripted, and the characters act autonomously, performing their roles in character. To ensure a user-centered experience, 


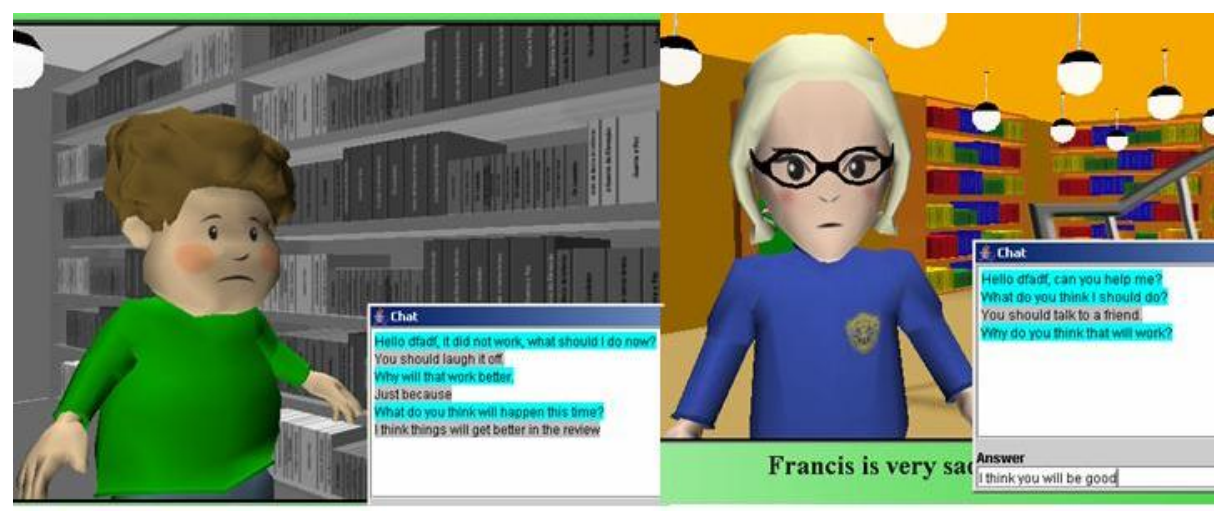

Figure 3: An interaction window between the victim character and the child (for the physical bullying and relational bullying)

the overall characteristics of each episode are decided by an external entity, a stage manager (see [25] for more details). This entity selects appropriate places and characters that potentiate the occurrence of certain events favouring an authored educational purpose. Nevertheless, the characters autonomously decide their actions.

Bullying, like many of our everyday problems, has no 'magic wand' solution - only more or less frequently successful strategies. The only universally accepted message is that passivity is no solution, and one should never suffer in silence. The purpose of the system is not to deliver a 'right answer' but to present a multitude of options to the child, and allow him or her to explore possible consequences for certain courses of action. The use of an intelligent virtual environment with characters and emergent narrative gives us that possibility.

\section{Empathy in Pedagogical Lifelike Characters}

The term "empathy" stems from Titchener [28], who derived it from the Greek "empatheia" which means "passion", "passionate affection" or "to be much affected" (Levy, 1997). Titchener used "empathy" as a translation of the German term "Einfühlung" which means "feeling into" somebody. Defined as "the capacity of participating in or vicarious experiencing of another's feeling, volitions, or ideas and sometimes another's movements to the point of executing bodily movements resembling his" [1]. This definition implies that, firstly, empathy is an internal state similar to an emotion; and secondly that emotional state can sometimes be recognised through imitative bodily movements. As reported by Plutnick [22] empathy is also found in animals and is a widespread phenomenon in the animal world, involved in a wide variety of behaviour patterns such as schooling or flocking. All these behaviours involve mimicry and affective communication. In general, empathy refers not to processes between a person and an object, but to processes between two persons, where one person perceives the 
other. The perceiving person, or the persons who "feels into" the other person, is called the "observer", and the perceived person is called "target".

Most contemporary psychologists agree that there are two aspects of empathy that have to be distinguished. The first one is the mediation of empathy and the second the outcome of the empathic process. Concerning the mediation of empathy, one can distinguish two different ways of mediating: (1) via the situation and (2) via emotional expressions. When empathy is mediated via the situation, the observer concludes the emotional state of the target from the situation the target is dealing with. For example, if the observer perceives the target being hit by another, he may think that he would be very angry in that situation himself. So the target will probably feel angry, too. Empathy may also be mediated via emotional expressions of the target. This occurs when the observer interprets the behaviour of the target, as for example, assuming that when a target smiles he/she is probably happy.

These two aspects give rise to the empathic process, which in turn may have an outcome. According to Davis [11] empathic process's outcomes can either be cognitive or affective. A cognitive outcome involves cognitive activity of the observer, such as obtaining more information about the target or acting to help the target, whereas an affective outcome (the one we usually consider as empathy) means that the observer experiences an emotion because of his/her perception of the target.

Our main focus in this paper is on how to build characters to evoke emotional responses (empathic responses) from the user. So, our main question is: How do we build characters that are able to establish empathic relations with the learners in Virtual Envrionments?

To address this problem, we have put forward an hypothesis based on the idea of proximity. There is evidence in literature that people experience more empathic emotions when the incidents are associated with people with whom they have a communal relationship (where communal relationships are friendship, romantic love or family relationship). Also, people who perceive themselves to be similar to another also perceive themselves as having stronger communal relationships with the other, and in turn, experience more empathic compassion when the other is in need. For example, if the target is of the same age and with similar features and attitudes. These findings suggest that one way for the user to feel empathy and put him/herself in the place of a character is to find similarities with between the user and the character, so that the user feels similar to the character. So, what we call the proximity factor, tells us that, in order for a synthetic character to evoke affective and cognitive empathy, users must feel close to the character. Further, we argue that this is achieved by designing the whole environment and situations in a way that users feel some degree of familiarity and closeness with the characters, environment and situations.

Based on this hypothesis in the design and character's creation of FearNot! we took several decisions where proximity was taken into the whole design process. Considering the two ways of mediating empathy we will address the problem considering:

- mediation of empathy via the situation which has lead to the creation of episodes, situations, behaviours and the environment that can lead to empathic reactions by the users;

- mediation of empathy via de emotional expressions has lead to the design and 
creation of the behaviours, physical aspects and emotional expressions of the characters.

From the start of the project we have involved children and teachers, and the characters were evaluated by the children from their creation. This evaluation was aimed at obtaining characters that children relate to and somehow are able to identify with. To do that, proximity was considered at several levels of design as described.

\section{Evoking empathy: The Situations and the Environ- ment}

Bullying is episodic, where a sequence of similar situations - sometimes apparently innocent on their own - builds into a serious issue that affects the victimized child. Such a build up of situations is essential for the development of empathy. It is essential that the child clearly acknowledges the situation as a bullying scenario, thus leading to an understanding of the unfolding events and to the establishment of a stronger empathic relation with the victim. To effectively achieve this, the early involvement of teachers and children in the development of storyboards became an essential task for the further progress of the project.

\subsection{Capturing patterns of bullying behaviour}

An essential aspect of the design and implementation of believable and interesting bullying scenarios concerns the profiles and roles designated for each character depicted within the scenarios. A number of research studies have been carried out to assess bullying profiles and a classification of distinct characteristics are evident for 'pure' bullies, 'pure' victim, bully/victim, bully/assistants, defenders and bystanders (e.g. [30]). The social characteristics of bullying behaviour have also been researched to ensure that the right contexts are implemented for the scenarios including gender issues, age, the role of peers in bullying behaviour, and bullying as a group process.

To do that, we used Kar2ouche which is a high fidelity storyboarding tool that allowed the creation of scenarios, populated with prototypical animated agents, that aimed to capture both direct and relational bullying behaviour taking into account the different group roles (bully, victim, bully/victim, defender, bully assistant). The storyboard scripts provided two different stories about direct and relational bullying devised by experienced psychologists with expertise in bullying research. Each story is comprised of a series of episodes/chapters (see Figure 4. The storyboards were used as an initial means of assessing character preference, engagement and empathy with the characters and different bullying roles.

Figure 4 illustrates clips from a direct and a relational bullying scenario. In the first clip we see the entire cast of a direct bullying situation, with a bully (Luke), a victim (John) and bully assistants in the background. In the second clip we see the victim (Frances) in a relational bullying situation with the bully (Sarah) and several assistants. Both stories begin with an introduction and background information about the protagonists. In the case of the direct bullying scenario this involves Luke (the 

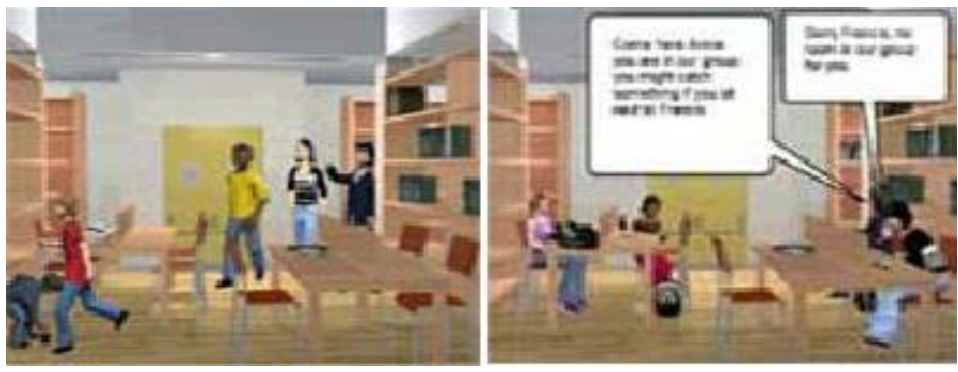

Figure 4: Storyboards done in the design of FearNot!

bully) knocking John's (the victim) pencil case onto the floor in the classroom and then pushing him off his chair when no one else is looking. Luke then verbally abuses John and tells him to stop being a wimp and threatens John that he better not tell anybody about the incident. This happens whilst the bullying assistants are egging Luke on. The story proceeds to show John trying out a number of different coping mechanisms including ignoring and trying to avoid Luke, fighting back with Luke and telling the teacher. The story illustrates ignoring Luke and fighting back as being unsuccessful strategies. The story ends with John telling the teacher and Luke being warned that if he did not stop bullying John, he would have to leave the school.

Several patterns of episodes, based on what we obtained from children, were constructed in Kartouche. To test their adequacy and truth to life, we performed some evaluation, where the children watched the direct and relational bullying scenarios and then completed a questionnaire comprised of both structured and semi-structured questions. Questions enquired firstly about the direct bullying scenario and secondly the relational bullying scenario. Questions about the child's empathic feelings towards the characters in both the scenarios followed. Direct Bullying Scenario Questions enquired about whether physical bullying happened at the respondents' school, whether they had experienced victimisation like 'John' the victim in the scenario and whether they had bullied anyone like 'Luke' the bully in the scenario. If children answered yes to experiencing or carrying out bullying they were asked to explain this in more detail. Children were asked whether the speech used in the scenario was realistic and similar to that used in their current school. Children then completed some questions about coping strategies they would employ if they were in John's (the victim) position and were asked to explain why they would select a particular strategy. Children were also asked what would be the worst thing to do to try and stop Luke bullying John. Finally, children were asked why they thought Luke bullied John.

Children were also asked whether they felt sorry for any of the characters and if so which characters and why, whether any of the characters made them feel angry and why and finally, how they felt overall after watching both the direct and relational bullying scenarios (very happy, quite happy, neither happy nor sad, quite sad and very sad).

Sample 80 children aged 9-11 with an average age of 9.7 years (SD: 0.66) participated in the present study involving two schools. One small rural school with children 
from middle to upper class socio-economic status, and one larger urban school with children from predominantly lower to middle social economic status participated. 43 boys and 37 females participated.

Children almost exclusively preferred the victims in the scenarios, with very few children expressing a preference for any of the three bully characters. However, there was a clear gender impact on most preferred character, with boys strongest preference being for the male victim, whilst girls were not as gender specific. Children least preferred the bullies, with a trend for boys to show least preference for the female bullying characters. Boys indicated least preference for both the female bully and the assistant. Girls were more evenly spread between the main bullies (Sarah and Luke) and few least preferred the bully assistant. This suggests that girls are able to distinguish between the severity of the role whereas the boys were focused more on gender rather than character activity. The majority of the participants when asked about 'prime character' wished to be a victim, but one that was the same gender as them. Notably, no boy expressed the desire to be a female bully and only 3 boys were prepared to be a female character of any sort. This has important consequences for the design of animated characters aimed at generating empathic relations. There is a need for focused scenarios to be developed which offers children same gender animated characters with whom to empathise. The situation seems to be more extreme with boys, who clearly find it difficult to empathise with a female character. This could be an effect of age, as in this middle school age group, girls are more socially and cognitively developed which may enable them to take on both gender perspectives and the different expressed behaviour patterns. The fact that most children would choose to be a victim over the bullies may be an indication of the story plot, with the victims working through to a successful outcome (i.e. the bullying stops).

These results were then used to impact the episodes and the characters built within FearNot!.

\subsection{Generating episodes in real time}

FearNot! can be seen as sequence of events in time that unfold bullying situations as a form of episodes. Given that explicitly scripting all the possible situations remains an unsurmountable task we need to make sure that the events generated by the application do follow the structure and patterns detected in the experiments done. Thus, the FearNot required a narrative control of some kind.

We can generically define a narrative as a sequence of events in time. Thus narrative management is needed to guarantee that the generated situations lead to the empathic relations we desire. We can structure narrative information through a hierarchy of levels of abstraction. Although we can create as many levels as desired, stemming from notions in drama arts, we envisioned the concepts of act, scene and beat. An act is the narrative's most abstract structure, representing significantly distinct sections of a narrative (eg., in the case of FearNot!, the final educational message is seen distinctively from the bullying situations). Each act can be seen as a set of scenes. Scenes within an act hold related content, but represent still distinct narrative moments. Finally, each scene contains a pattern of beats that are the most basic elements within the narrative. A beat describes event patterns that are relevant for the narrative within the 
scene where they are active. An event signals some change in the world, usually caused by the actions of the characters. Events are very low-level and are not considered at the narrative level, although they can satisfy a pattern that constitutes a beat. A beat can detect either just a simple event (e.g., a specific action) or a complex pattern of events (e.g., a particular sequence of actions).

Building from these base concepts, bullying situations arise as specific instances of narrative building blocks (acts, scenes and beats) using specific concepts. Such knowledge is clearly domain-dependent and as such it must be drawn from experts in the field through the use of knowledge elicitation tools (as we have seen in previous section). This knowledge consists in specific nomenclature of concepts and the description of their properties and relations. A victim, as a character role, is an example of a concept of the bullying domain. Narrative information can be described using a simple rule-based system, therefore easing the use of the elicited knowledge.

Nonetheless, long-term and abstract planning of a whole situation is extremely complex. In a long-term, to build a complete situation, one would need to integrate in the narrative all possible behavior traits that characterize each role, otherwise failing to comply to behaviour believability. Following an agent-based approach, the use of autonomous characters distributes this complexity thus enacting emergent narratives. Although the situation is externally prepared, the characters autonomously decide their actions, performing their roles in character (see next section for more detail on the characters behaviours). For example, if we wish to potentiate a direct physical bullying event, we can choose a situation involving the bully and the victim alone in the dressing room. If some aggression is detected, the episode then halts and the system passes to the reflection phase (where the child advises the victim).

Taking on ideas from role playing games, we can identify several levels of intervention for narrative management[16]. To provide for a balance between author-induced content and user's free-play, narrative control can be carried at the levels of simulation and presentation [26].

At the simulation level, the narrative only controls the episode (scene) settings by placing the objects and characters in appropriate locales and sending a play command, letting the characters play their role within that episode. This is clearly the solution that enables more interactivity and variability, because its results depend on the character agent architecture and even on the user's input (if the user can intervene). To control the execution of the episode, the episode's beats are used to detect expected event patterns. When a beat event pattern is detected, its associated rules are executed or/and other beats are activated to listen for other event patterns. Associated rules may include the episode's termination (in a successful way) or indicate a counter-measure to avoid unintended pathways. These counter-measures usually include refining the level of control.

The stage manager can directly control the characters by sending them orders (for example, to force an action that is necessary for a specific event pattern to succeed). This is a dangerous level because not only the view actions are not finely described (i.e., there is still the danger that exists at simulation level of a lack of control of the presentation), but it also requires a strong knowledge of 
the character roles, to avoid unbelievable behaviour. It also makes interactivity difficult, although not as hard as at the presentation level.

At the presentation level, the narrative can be described as a linear sequence (a script) of view actions. This is the level that produces the best visual results, but requires much more work, and is completely inflexible in terms of enabling interactivity. The presentation level is free of domain, including view actions like "play animation", "play sound", "move object", "zoom camera". This level works as if the stage manager is directly communicating with the view manager, while the virtual space is not being used (the character agents are actually paused). In this case, the stage manager will order (through the execution of an effector) the view manager to execute a script, in the appropriate view action language.

A particular user experience will consist on a traversal of the narrative content structure. The stage manager is therefore used to guide such a traversal, using an appropriate level of intervention. A first introductory act is composed of a single scripted scene, depicting the introduction of the school environment, the characters and the situation. Similarly, a final message act displays an educational message. In this case, though, the particular script presented depends on what happened previously. The impact of bullying events is greater if the user knows the characters. Since we wish to favour the development of an empathic relationship, certain situations (mainly initially) must increase the feeling of proximity. The main act constitutes the bullying scenario itself. It starts with an initiating bullying episode that introduces the child to the problem that is occurring. In this episode, a bullying incident is absolutely essential for the rest of the interaction. Other acts follow, similar in nature but different in function. This entity uses rule-based authored (by experts in bullying) knowledge, this way selecting appropriate places and characters that potentiate the occurrence of certain events favoring specific authored purposes, drawing on situations that we clearly find in schools.

Each simulated episode within the bullying scenario act defines a set of encounters that enacts (rather than dictate) bullying situations. Each encounter is emerging, and is defined in a way that autonomous characters, if designed according to the roles they play, should effectuate the expected situation, although always in a different way, according to a multitude of factors. Nevertheless, control mechanisms overlook at the emerging behaviour and can take counter-measures to force a pedagogically accepted turn of events. This notion of encounters [16]) led to the creation of the meta-scene, which is an abstract scene that needs to be instantiated with appropriate data. This concept was used in the (non)-bullying scene 2, which, according to appropriate initial facts, can become an episode where no bullying incidents happen or one that such incidents happen. For example, to create a non-bullying episode, we can start without the bully and/or bully helpers.

\subsection{Evoking empathy: The environment}

As with the characters we have designed a set of schools (although all are cartoon like) that resemble to different types of schools the children belong. So, for example we have urban and countryside schools as well as country specific schools. For example, 
Figure 3 shows one character in a urban Portuguese school, and 5 shows one of the designed classrooms.

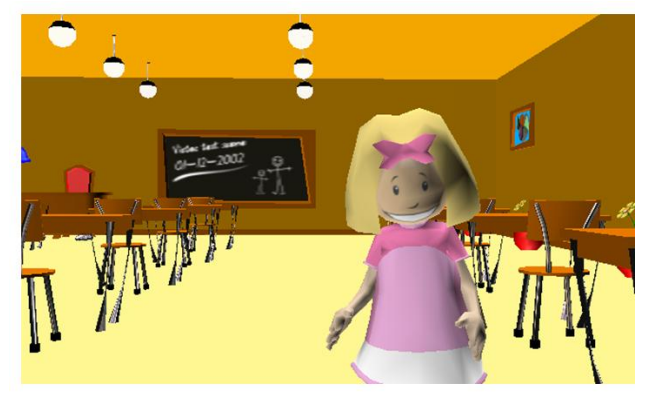

Figure 5: One of the characters in a classroom

\section{Evoking empathy in FearNot!: The character's be- haviour}

Characters must act in a believable way. So their actions must be generated in a way that patterns of behaviour allow for children to recognise as common behaviours found in school's pupils. Given that we didn't want to script the actions of the characters, the architecture developed for our FearNot! synthetic characters allows for a dynamic generation of actions in a believable and autonomous way.

However, to achieve a degree of believability and empathy neede, the architecture developed must contain a way to trigger emotional states in the characters (which in turn will lead to emotional expression- a mediator of empathy). Thus, we developed an emotional module which is responsible for appraising the situations in a scene and activate emotional states, which in turn will lead to action tendencies and coping strategies. The architecture also contains a representation of others, in particular their emotional state and an action selection mechanism that leads the characters to act according to a certain emotional state. The main aspects of this architecture are thus:

- A model of the world that includes a model of the self with emotions representation and a model of the other agents (also an affective model);

- The emotional model is parameterized for agent based in a personality profile (see below);

- An appraisal component;

- An action selection component that depends on action tendencies associated with the emotions represented; 
- A coping mechanism;

- An affective expression component including body, facial expressions and speech.

Each agent in the world (a character such as Luke or John) perceives the environment, through a set of sensors (allowing the perception of events, objects, etc. in the world) and acts on the environment though its effectors, allowing different actions to be performed. For example, a bully may hit the victim and the victim may cry. The agent architecture is shown in Figure 6.

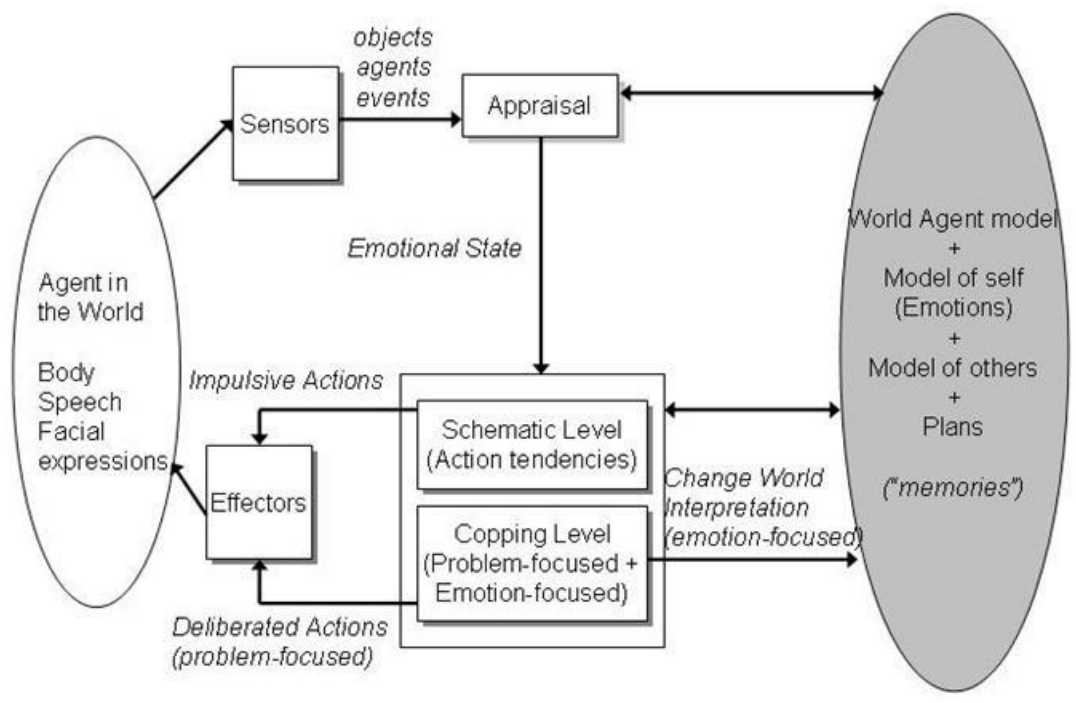

Figure 6: Agent Architecture Diagram

Upon receiving a perception (which can, for example, be the presence of another agent or an object, or even an action from another agent) the agent appraises its significance and triggers the appropriate emotions. Additionally, if a goal has become active, it will add a new intention to achieve the active goal. Intentions map directly the concept of intentions in a BDI (Beliefs-Desires-Intentions) agent architecture [31]. They represent the agent endeavor to act in order to achieve the desired state.

After the appraisal process, it is necessary to choose the most adequate action. For successful adaptation to the environment, emotions must have an effect on the actions of the characters. For example, if our agent is sad it will act differently from a situation when he is happy. However, the resulting actions are not always of the same type. Lazarus [13] states that action tendencies are innate biological impulses, while coping "is a much more complex, deliberate and often planful psychological process". This distinction also exists in characters' actions in FearNot!. For example, if the Victim 
character starts to cry when she is bullied, it is not because she has a goal that involves crying. In fact, after this she will feel ashamed for crying instead of fighting back. The victim crying is modelled as an innate reaction to a particular distressed emotional state and the inability for fighting back. On the other hand, other actions, such as talking to someone is a planned actions resulting from the internal goals of the agent.

Following the same ideas, the action selection mechanism in the FearNot! agents is composed by two layers. The first one is the schematic layer where a set of action tendencies triggered by particular emotions is defined. For instance, if the bully character gets very angry, it will tend to kick everything in his path. A second layer is the conceptual/coping layer where two kinds of coping are defined. The first is problem-focused coping, where the character tries to plan and act to achieve his goals; and the second is emotion-focused coping that works by altering the character's interpretation of the environment. For example, an agent that feels distressed for not being able to achieve a given goal, may generated emotion-focused coping by lowering the goal's importance and thus reducing his distress. In this way, emotions will not only influence the agents' reactive behavior, but also guide the planning process, since emotional focused coping changes the agents interpretation of its plans.

A description of these processes involved in the agent's minds is provided next.

\subsection{Appraisal}

The emotional component of the architecture is based in Ortony, Clore and Collins Theory of Emotions [20]. The use of OCC for the appraisal, by contrast with other emotion architectures, allowed us to easily and pragmatically obtain patterns of behaviours in the characters (thus allowing for the creation of a bully or a victim in an easy way). Thus, emotions are seen as valenced reactions to an event in the world. The character's emotions are triggered by an appraisal process, which can be seen as a subjective evaluation of a given event according to the character goals, standards and beliefs.

Our model uses two of OCC defined goal types, which are the active-pursuit goals and interest goals. The active-pursuit goals are goals that the characters actively try to achieve, like going to watch a football much in the school. Interest goals represent goals that a character has but does not pursue, such as, for instance, wanting his favourite team to win a match, or avoiding get hurt.

As proposed by Martinho [18], part of the triggering of emotions is handled by a set of emotional reactions rules. An emotional reaction rule is composed by a domain specific construal frame extended with values for some of OCC emotion intensity variables. The following figure shows three examples of these rules.

As seen on the examples, a reaction rule is composed by the event information and the values for the appraisal variables. The first part is used to match the rule against the perceived events, for instance the leftmost rule is selected if the event corresponds to a cry action made by another character. The second part determines which emotions are generated and the corresponding intensity.

Emotions can also be triggered by events that affect the plans the agent is pursuing. However, instead of writing domain specific reaction rules to handle prospect based reactions (as was done by [18], in this architecture we followed a similar approach to 

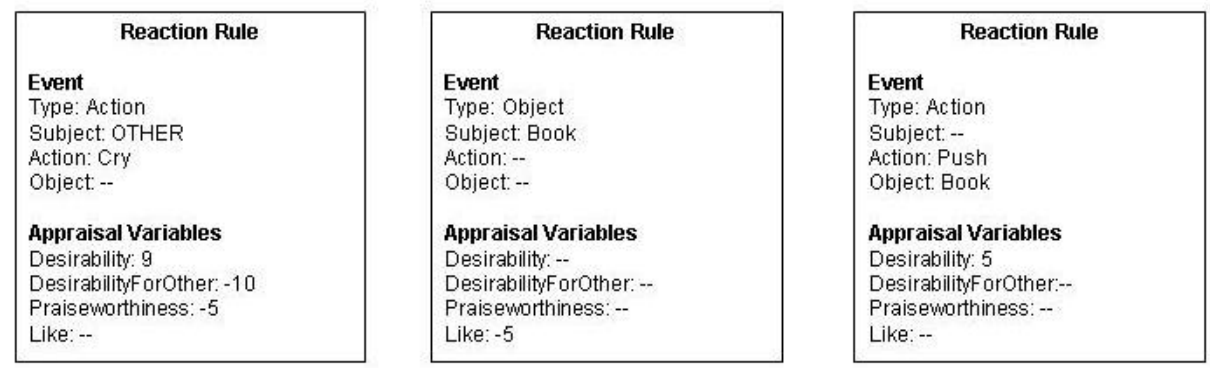

Figure 7: Emotional Reaction Rules

the one used in the mile System [9] taking advantage of explicitly storing the agent plans state and intentions into memory. With this approach, prospect based reactions can be automatically obtained from the plans and goals active in the agent memory.

\subsubsection{Emotion Generation}

Each time an emotion is created, a potential value for the emotion is determined from the event appraisal. However, emotions do not become active automatically. Each character has a set of emotional thresholds and emotional decay rates (one for each emotion type) according to his personality. The threshold represents the character resistance towards an emotion type. The decay rate represents how fast the emotions of an emotion type fade out. When the emotion intensity reaches zero it is removed from the character emotional state. An emotion is added to the character emotional state only if the emotion potential surpasses the defined threshold.

\subsection{Action Selection}

As mentioned before, the action selection mechanism is composed by two layers, the schematic and the coping level. Since the schematic level defines action tendencies, which represent innate reactions to the environment, they have priority over the Coping level actions and thus are immediately executed. For example, if the bully most intense emotion is Gloating at the victim (happy about something bad happening to the victim) he will mock him (action).

\subsubsection{Schematic Layer}

The Schematic Layer implements the characters action tendencies. It consists on a set of actions that are available according to the character's emotional state.

The action selection mechanism starts by determining which actions can be executed, by checking its preconditions. Afterwards it selects the action triggered by the most intense emotion the character is experiencing. If the emotion intensity is greater than the specified minimum, the action is executed. If more than one action rule is selected (triggered by the same emotion), the most specific one is preferred. 


\subsubsection{Conceptual Layer}

A continuous planner [24] that uses partial-ordered-plans builds up the core of the conceptual layer. After the appraisal process, the planner selects the currently most intense intention from the intention structure. The selected intention becomes the target goal which the planner will try to achieve. Afterwards, the continuous planner removes a flaw or executes an action (if the plan is complete). The resulting plan is stored with the intention, so that it can be continued later on.

A continuous planner works by incrementally building, executing, and monitoring a given plan. Since the planner continuously monitors the environment, it detects when an action is accomplished or fails. It can handle unexpected events that affect future plans and it can handle serendipity. Suppose that the planner has finished building a plan to achieve a goal, if some other agent comes in and achieves some precondition for us, the planner will detect that the condition holds true in the start step and will remove the action used to achieve such precondition.

As the planner builds a way to achieve the goal, more than one different plan may be construed. For instance, when removing an open condition, there may be more than one alternative to achieve the condition (two different actions). Neither of the alternatives can be forgotten without taking the risk of not finding the best course of action, or even to find any solution whatsoever. So, instead of one single resulting plan, it is necessary to store all alternative plans. Taking this into account, the planner must select one from all alternative plans in order to continue planning or execution.

\subsubsection{Plan Selection}

In FearNot!, plans representation is based on classical planning and decision theory. FearNot! generates Partially Ordered Plans which are modeled as a set of operators and additional constraints. These operators are a slight modification of STRIPS operators, associating probability values to the effects and represent the actions that an agent may take in the world.

An action or effect probability is obtained from two sources: the character experience, whenever the character does some action it remembers the number of times it was successful; and an interpretational bias. This bias allows the character to use emotional-focused coping to change the subjective probability that a given effect occurs.

After selecting the intention, the planner must also determine what the best plan to continue the planning process is. We could select the one that seems more probable, but unfortunately this method would generate a breath-first search on the plan space. Since adding a step only decreases or maintains the plan probability, the planner will select first plans with fewer steps (but also farther from the goal). Therefore we would like that the planner also look at the number of open preconditions (how far is planner of building a solution?). So it was used a heuristic function that looks at these parameters. The plan chosen for processing is the one with the lowest value of $h$.

$$
h(\text { plan })=(\text { numberOfSteps }+ \text { numberOfOpenPreconditions }) / P(\text { plan })
$$


The mechanism described so far corresponds to Problem Focused Coping, which focus on acting on the environment (using planning abilities).

\subsubsection{Emotion Focused Coping}

Emotion Focused Coping works by altering the character's interpretation of circumstances (for instance the probability bias of a particular effect). Notice, that by changing the interpretation, the appraisal process will generate distinct or weaker emotions, thus allowing strong negative emotions do fade out and eventually disappear. Additionally, this interpretation change will affect the planning process.

Emotion Focused Strategies used in FearNot! are similar to the ones described in Marsella and Gratch work [17]. The selection of a coping strategy is a two stage process: first a coping opportunity is identified generating possible coping strategies; and finally a coping potential is determined and used to decide if the strategy will be used. All the generated strategies can be combined and applied at the same time. The several emotion focused strategies will be described next.

Acceptance: Acceptance is the recognition that a goal is not possible to achieve or protect. This strategy is generated on two distinct situations. When the planner cannot build a plan to achieve the goal, the intention is removed and the agent accepts the goal failure. However, this strategy is generated even before the planner tries all possibilities, if the goal probability goes below a defined threshold, then an acceptance strategy is triggered. The agent starts to consider the hypothesis of giving up the goal.

The other situation happens when it is not possible to achieve a goal without violating an Interest Goal. In this situation the protection constraint is removed from the plan so that the planner can introduce an action that violates the condition but achieves a needed precondition. This strategy is applied if the emotion generated by the failure of the active-pursuit goal is of greater intensity than the emotion generated by the failure of the interest goal.

Denial / Wishful Thinking Denial works by denying the reality of an event. It is used when an event/action has an undesirable effect, whether it is an effect that threatens a causal link, and no promotion or demotion resolves the threat, or an effect that spoils a protection constraint of an Interest Goal. This strategy works by lowering the effect probability so that the effect can be ignored. This strategy is always applied if the effect probability is not too high.

Mental Disengagement When a desired goal seems unachievable, mental disengagement works by reducing the agents "investment" into the goal, i.e. the goal importance is reduced. This strategy is selected if the character most intense emotion is Fear for not achieving the goal. Since the character does not want to feel Fear, one way of reducing it is to reduce its importance of failure. Given that the prospect based emotions are automatically determining by the plan probability and importance, lowering the plan importance will immediately lower the intensity of the Fear emotion. 
Note that these strategies will also indirectly influence the planning process. By changing goals importance, we may change the next intention to be selected by the planner. By changing effects probability we are changing plan probability and therefore a different plan can be chosen next to continue the refinement process. Also, changing these parameters will lead to reappraisal that will generate different emotions, which will change the planning process, and thus generating new coping strategies which will once more lead to a new appraisal, and so forth.

\subsection{Illustrative Example}

In order to understand the entire architecture, let's examine a small example. In one of FearNot's episodes, John (the victim) is sitting quietly in the classroom before the class commences. The bully character, Luke, walks in and decides to push the victim books to the floor, teasing him to get them (see Figure ??). When John gets up to pick his books, he is pushed by Luke and falls. John starts to cry endlessly while Luke gloats and threatens him.

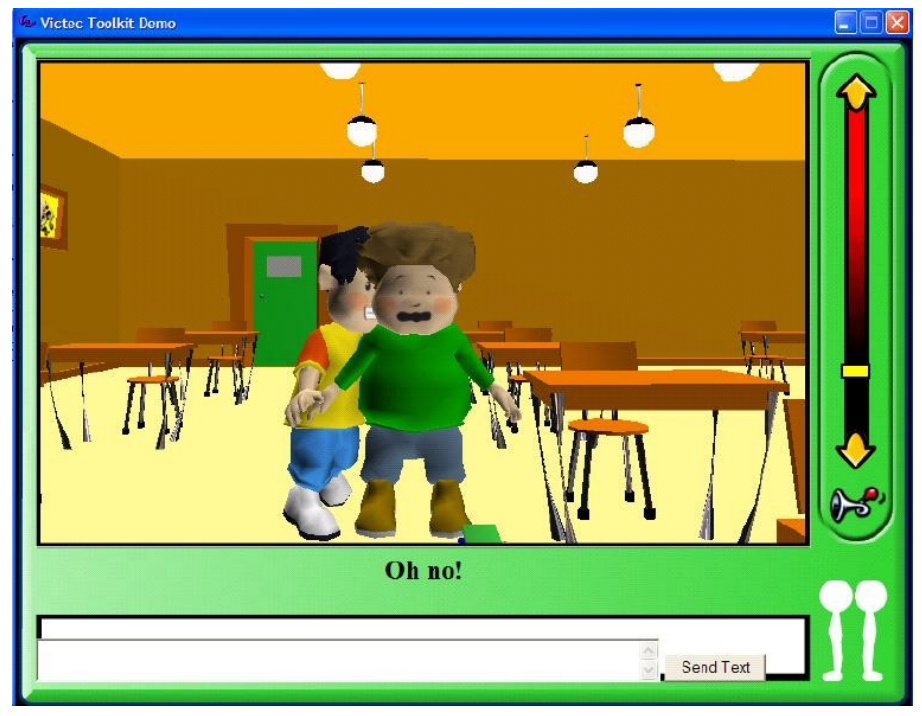

Figure 8: Illustrative Example

In terms of what happens in the character's minds, the first important event, when the bully enters the classroom, he will receive perceptions from the world showing that the victim and books are there. These perceptions trigger Luke's active-pursuit goal of bullying any of the victim's props. As the goal becomes active (see previous secions), an intention to achieve it is added to the conceptual layer. As this goal is quite important to the bully, the intention draws Luke's attention by generating a strong Hope emotion, and therefore, being the most intense intention (and the only one for the moment). This leads Luke to start building a plan in his "mind" to accomplish that goal. 
The plan is built very quickly, and Luke soon realizes that the plan is very likely to succeed, thus reinforcing his hope emotion and his intention to do it. After the plan is complete, Luke starts to execute it: he walks near the victim and pushes the books on the table. The push event is appraised differently by the two characters. As shown in the Figure 9, Luke has an emotional reaction rule specifying that such event is very desirable to him and undesirable to the victim, and also he considers it praiseworthy. Therefore, he will feel Joy, Gloating, Pride and Gratification. Additionally, the event is appraised regarding his goals and given the bully goal is achieved, he will also feel Satisfaction.

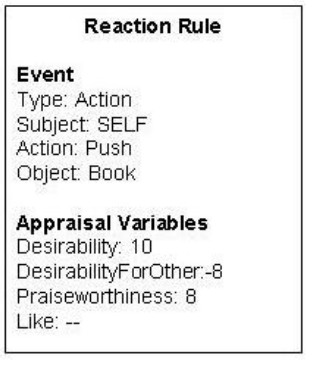

a) Luke's Reaction to the push book event

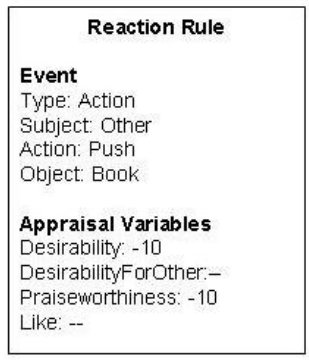

b) John's Reaction to the push book event

Figure 9: Example

John, on the other hand will feel Distress, Reproach and Anger. But, more importantly, the event causes an active-pursuit goal to become active. John does not like to have his things on the floor, so he adds an intention to pick his books up and return them to the table. So following the same process described above, he will build a small plan to achieve such end. At the same time John is building his plan, Luke's emotional state will trigger an action tendency. He has an action rule specifying that when he feels satisfied about bullying a victim's object (pushing it, stealing it, etc) he will tend to tease the victim. So, Luke will start a Tease "Speech Act", saying something like: "come and get them, you muppet!".

When John gets up and moves near the books, Luke sees a new opportunity to bully the victim, so a new active-pursuit goal becomes active. This time, the goal is to physically harass the victim. Once more the plan is built very quickly (since it is such a small plan), and generates the same emotions as above. Therefore, at the same time John is trying to pick his book up, Luke pushes him and makes him fall.

Like before, this event is appraised by the two characters. Luke feels even more Joy, Gloating, etc and also Satisfaction for achieving his goal. At this moment, the Gloating emotion has a quite high intensity thus triggering another action tendency. When the gloating emotion surpasses a defined threshold, the bully starts to perform Gloat/Mock Speech Acts. On the other hand the victim appraises the event regarding his goals, and this particular event seems to thwart two of his goals: the active-pursuit goal of getting his books, and an interest goal of being healthy (or not getting hurt). 
Besides feeling disappointed, John distress becomes incredibly high (remember he was already feeling distressed), and triggers his action tendency to cry.

At this stage, John's mind can use emotion focused coping to mitigate its negative emotions. Since the goal of getting his books seems unlikely for the moment, John uses a mental disengagement strategy to lower the importance of this goal. By lowering the goal's importance, the intensity of the Fear emotion (for not achieving the goal) is also reduced. On the other hand, John uses a denial/wishful strategy to lower the probability that the push action has the effect of hurting him. Such probability change leads to a different appraisal of the threat to John interest goal of being healthy, which seems weaker and more unlikely, and thus reducing his distress. In other words, John starts to think something like "Well, he did not hurt me after all." or "it did not hurt that much" and slowly calms down and stops crying.

On the other hand, the crying action is appraised once more by both characters, repeating the same processes described above. Finally, the episode ends with Luke threatening John to not tell anyone (or to stop crying) and with poor John feeling very ashamed for crying and not doing anything.

\section{Evoking empathy: The character's physical aspects}

In FearNot! characters are 3D embodied characters, which means that we can use facial expressions, attitudes, body expressions to convey their emotional states.

In order to design the characters we made some preliminar tests with children and designed two types of characters: realistic versus cartoon like. Although at first, and according to the proximity argument, we should adopt realistic characters it was clear from the studies and results that learners of this age preferred the cartoon characters (see Evaluation section). Inspired by the very popular characters from a Portuguese children's web portal (Cidade da Malta in http://www.cidadedamalta.pt/) originally in $2 \mathrm{D}$, we have converted the characters into $3 \mathrm{D}$ and adopted them adequately for the age and gender groups involved.

Furthermore, the characters and the situations for the age groups we are targeting range a quite distinct set of children's appearances (see a set of characters for the UK version in Figure 12) so that children can easily identify with one or another character

For each country (UK, Portugal and Germany), we designed different characters given that children in the UK have uniforms and in Portugal and Germany do not.

We also have considered specific situations for both genders (more direct bullying for boys and relational bullying for girls, see Figure 11).

\subsection{Evoking empathy: Conversations and Expressions}

Further, and as described earlier, empathy can be mediated in an affective way, so the characters must be able to express emotions in facial expressions, voice and body posture. If the user perceives the agent expressing emotions that are adequate to the displayed situation, believability and empathy should increase. In FearNot! we use 


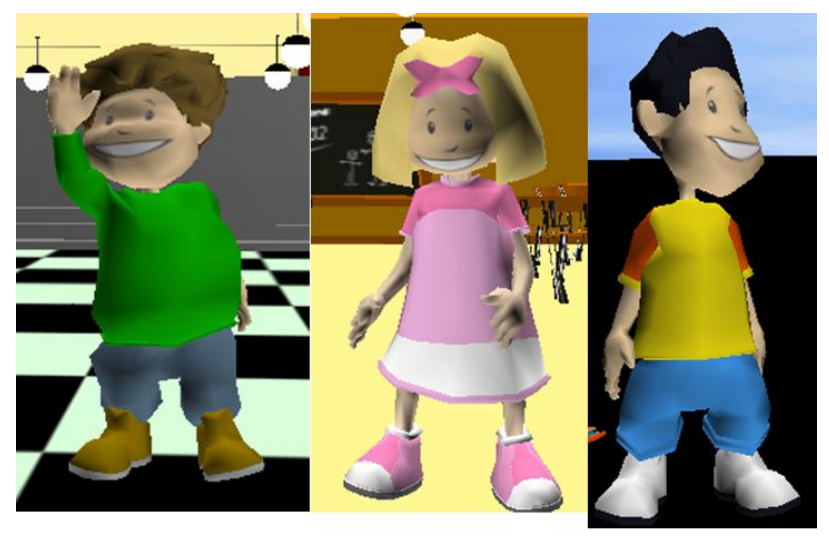

Figure 10: Three of the characters developed for the FearNot! application (John, the victim, Martinha, the neutral and Luke, the bully)

mainly facial and body expression. A precondition therefore is that the emotional expression can be recognized by the user correctly. Another possible mode of emotional expression that avoids the danger of misinterpretation is language. The agent could inform the user about his emotional state verbally. One should note that the cognitive component of empathy would be realized if the user has the impression that the virtual agent "knows" something about the user's inner state.

In order to clearly convey the character's emotional state it was easier and more effective to adopt cartoon like characters. In fact, tests carried out with children in associated schools revealed that children preferred the cartoon characters. This also reduces the importance of using complex and resource intensive real-time facial animation and lip-sync. Simple textured faces (see Figures 10 and 13) can be very believable (even more believable than perfectly modelled faces).

As for conversations, we collected from schools several scenarios with typical dialogues and even with common aggressive names uttered between children of the targeting age. These dialogues are used as patterns for the language generation system. 


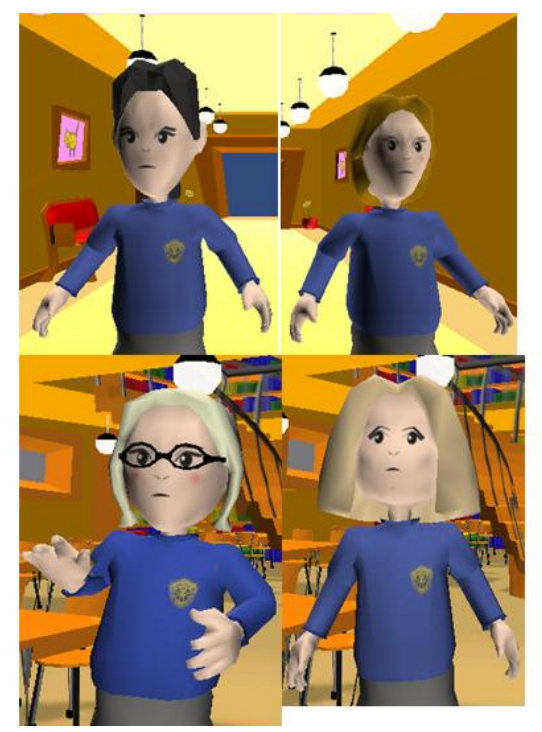

Figure 11: Girls characters for the relational bullying scenarios

\section{Evaluation and Results}

The main focus of this evaluation was to consider the different perspectives and empathic reactions of adult and child populations with the system. The main questions we were seeking to answer were: Are there differences in the views, opinions and attitudes of children and adults? And, if there are differences in the empathic reactions to the system and do these have any important design implications for empathic embodied characters?

\subsection{Experimental Design}

Using one limited version featuring a single bullying episode of FearNot! already released and evaluated with several types of users, we have conducted a set of experiments in three different countries: UK, Portugal and Germany. All the main aspects of the architecture were already in place and the characters built follow the requirements presented. Children were shown the trailer of the FearNot! which depicts one physical bullying episode developed by experts in bullying research in conjunction with teachers and pupils. All characters and animations, places and objects were transposed to 3D by the team of designers according to the principles just described. The dialogues were obtained through the recording of real voices. The physical bullying episode contains 3 characters, Luke the bully, John the victim and Martinha the narrator. The trailer begins with an introduction to the main characters, Luke and John and subsequently shows Luke knocking John's pencil case off the table and then kicking him to the floor. John then asks the user what he should do to try and stop Luke bullying him and arrives 


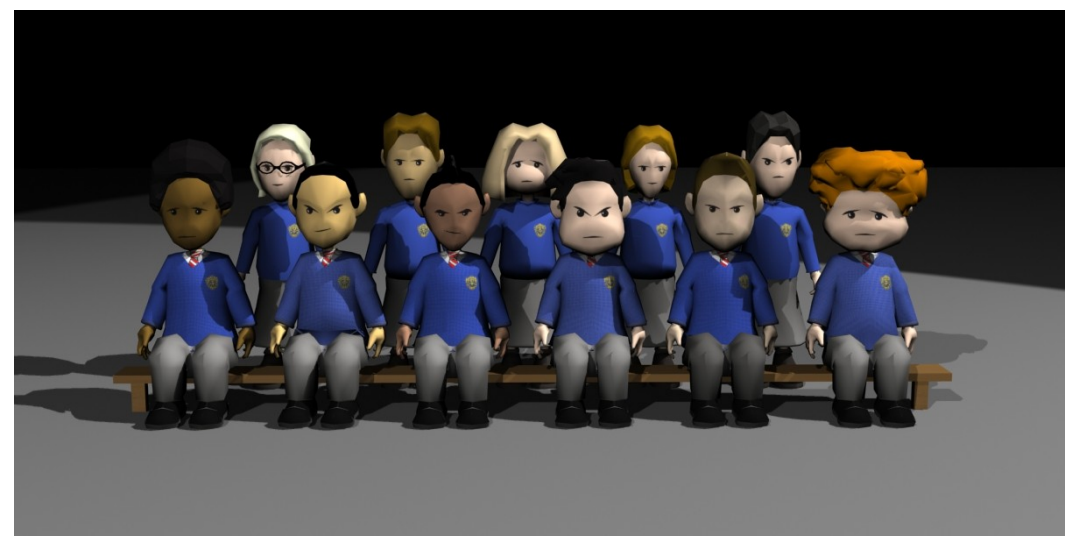

Figure 12: The students developed for the UK schools

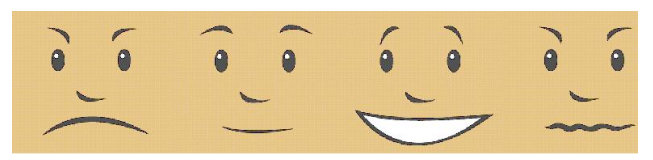

Figure 13: Example of Some Facial Expressions in Characters

at 3 possible choices: 1) Ignore Luke, 2) Fight back, 3) Tell someone that he trusts such as his teacher or parents. Developmental constraints of the application did not allow us to include the dialogue phase in the first trailer developed. Nonetheless, the importance of the dialogue phase for the overall success of the application required us to include it in the demonstrator (as briefly stated).

A questionnaire applicable for children and adults was designed in order to evaluate aspects of FearNot!, the VICTEC bullying demonstrator. The questionnaire was divided into 7 main sections and was predominantly measured according to a 5 point Likert scale. Table 1 illustrates the main sections of the questionnaire and the nature of the questions within each section. 


\begin{tabular}{|c|l|}
\hline Section & Nature of question \\
\hline \hline $\mathbf{1}$ & $\begin{array}{l}\text { Preference for cartoon or } \\
\text { realistic characters }\end{array}$ \\
\hline $\mathbf{2}$ & $\begin{array}{l}\text { Characters attributes } \\
\text { - voice believability } \\
\text { - likeableness } \\
\text { - conversational content } \\
\text { (believable to unbelievable) } \\
\text { - conversation interest (interesting or boring) } \\
\text { - realism of characters (true to life to false) }\end{array}$ \\
\hline $\mathbf{3}$ & $\begin{array}{l}\text { Character Movement } \\
\text { - movement believability (believable to } \\
\text { unbelievable) } \\
\text { - realism to movement (realistic to unrealistic) } \\
\text { - smoothness of movement (smooth or jerky) }\end{array}$ \\
\hline $\mathbf{4}$ & $\begin{array}{l}\text { Appearance of the school environment } \\
\text { Bullying Storyline } \\
\text { - storyline believability (believable to } \\
\text { - unbelievable) } \\
\text { - storyline lenght }\end{array}$ \\
\hline $\mathbf{6}$ & $\begin{array}{l}\text { Character preference } \\
\text { (character likes most and character liked least) }\end{array}$ \\
\hline $\mathbf{7}$ & $\begin{array}{l}\text { Empathy towards characters } \\
\text { Feeling sorry for characters } \\
\text { (and if yes which character) } \\
\text { Feeling angry towards the characters } \\
\text { (and if yes which character) }\end{array}$ \\
\hline
\end{tabular}

Two hundred and twenty five questionaires were done, out of which 128 by children from schools in England and Portugal. The remaining were done by adults (teachers and experts). These are some of the results (more on these results can be found in [10]) that show partially the significance of the proximity factor.

\subsection{School Environment}

Significant differences were revealed between child's, expert's and teacher's views of the appearance of the school environment in the trailer. Post hoc tests showed that these significant differences were between the child and expert views and between the child and teacher views for the attractiveness of the school environment, indicating that the children viewed the school environment more positively than experts and teachers. Furthermore, there were also significant differences between child, expert and teacher views in relation to the match between the environment and the characters. These significant differences lay between the teacher and the child, where children were significantly more positive towards the match between the school environment and characters compared to teachers. We found this result quite positive as we wanted children to feel 
as close as possible with the environment.

\subsection{Character Movement}

Concerning the character's movement, there were significant differences between the stakeholder groups and views of the believability of character movement. Children thought that the character movement was significantly more believable than teachers, which again is quite positive. Overall, no significant gender differences were revealed for the believability of character movement, however, when age was taken into account, female children found character movement significantly more believable compared to female adults who found the character movement least believable. Again this show that our design was able to inspire more belivability to the right target users. Significant differences emerged for views of the realism of character movement where children thought that the character movement was significantly more realistic compared to teachers and experts. An independent samples T-test revealed significant gender differences for the realism of character movement. Females found character movement significantly more realistic than males. When age was considered, female children found the character movement significantly more realistic compared to male children, male adults and female adults.

\subsection{Conversation and Storyline}

Significant differences were found in the views of the true-to-lifeness of character conversation, where teachers found the character conversation significantly more false and less true to life compared to children. Given our effort in obtaining real children dialogues and scenarios, this again is a quite positive result. Furthermore, significant differences were found between groups for views of the believability of the storyline where children found the storyline significantly more believable than teachers.

\subsection{Affective and Cognitive Empathy}

Significant differences were found between children, experts and teachers for affective empathy. Significantly more children $(80 \%)$ expressed feeling sorry for the characters compared to teachers and experts (70\%). Affective empathy was only expressed for Luke and John, and not for Martinha. Significant differences were uncovered for age and gender, $(x=15.02, N=213, \mathrm{df}=3, \mathrm{p}=0.002)$ where significantly more female children (95\%) expressed affective empathy compared to male adults (67\%).

Significant differences were found between children, experts and teachers for cognitive empathy. Significantly more children $(71 \%)$ expressed cognitive empathy towards characters compared to experts (47\%) and teachers (28\%). Significantly more experts expressed anger towards John (the victim) compared to children and teachers and significantly more teachers expressed anger towards Martinha compared to experts and children. Interesting significant age and gender differences emerged, where significantly more female children expressed anger towards the characters compared to adults. This anger was almost exclusively directed at Luke (90\%), the bully, which 
again is a very positive result because it shows that FearNot! can evoke emotions to children of the age that we are targeting.

\section{Final Discussion}

In this paper we have provided a discussion on some of the features needed to build characters that are able to establish an empathic relation with users. One of the issues here described is what we call the proximity factor that tells us that, in order for a synthetic character to evoke affective and cognitive empathy, users must feel close to the character. Further, we argue that this is achieved by designing the whole environment and situations in a way that users feel some degree of familiarity and closeness with the characters, environment and situations.

To illustrate these issues we have presented a system FearNot! that has been developed to address bullying problems in schools using empathic synthetic characters. We have described some design decisions of FearNot! and discussed the results attained with the trailer, in particular the aspects associated with the proximity of the characters with the user.

\section{Acknowledgements}

The work here reported is part of the VICTEC project. We would like to thank all the partners in the project for their contributions in some of the issues here reported.

\section{References}

[1] Webster's Third New International Dictionary. 1971.

[2] M. Barnett. Empathy and related responses in children. In Empathy and its Development. Cambridge University Press, 1987.

[3] J. Bates. The role of emotion in believable agents. Technical Report CMU-CS94-13, Carnegie Mellon University, 1994.

[4] R. Bindiganavale, W. Schuler, J. Allbeck, N. Badler, A. Joshi, and M. Palmer. Dynamically altering agent behaviors using natural language instructions. In Procedings of the International Conference on Autonomous Agents (AA 2000). ACM Press, 2000.

[5] A. Boal. Legislative theatre: Using performance to make politics. Routledge, 1999.

[6] K. Bryant. An index of empathy for children and adolescents. Child Development, 53, 1982. 
[7] J. Cassell. Nudge nudge wink wink: Elements of face-to-face conversation for embodied conversational agents. In e. a. J. Cassell, editor, Embodied Conversational Agents. MIT Press, Cambridge, MA, 1999.

[8] K. Dautanhahn and S. Woods. Possible connections between bullying behaviour, empathy and imitation. Technical report, 2003.

[9] J. Gratch. Emile: Marshalling passions in training and education. In Autonomous Agents 2000. ACM Press, 2000.

[10] L. Hall, S. Woods, K. Dautenhahn, D. Sobral, A. Paiva, D. Wolke, and L. Newall. Designing empathic agents: Adults vs. kids. In Intelligent Tutoring Systems. Springer, 2004.

[11] M. H.Davis. Empathy: a social psychological approach. Dubuque: Brown and Benchmark Publishers, 1994.

[12] R. J. and J. L. Integrating pedagogical capabilities in a virtual environment agent. In L. Johnson and B. Hayes-Roth, editors, Autonomous Agent '97. ACM Press, 1997.

[13] R. Lazarus. Emotion and Adaptation. Oxford University Press, 1991.

[14] J. Lester, S. Converse, S. Kahler, T. Barlow, and R. Bhoga. The persona effect. In Proceedings of Computer Human Interaction'97. ACM Press, 1998.

[15] J. Lester, S. Towns, C. Callaway, J. Voerman, and P. FitzGerald. Deictic and emotive communication in animated pedagogical agents. In C. J., P. S., and S. J., editors, Embodied Conversational Agents, Cambridge, USA, 2000. MIT Press.

[16] S. Louchart and R. Aylett. Narrative theory and emergent interactive narrative. In Proceedings of 2 nd International Workshop on Narrative and Interactive Learning Environments, 2002.

[17] S. Marsella and J. Gratch. Modeling coping behavior in virtual humans: don't worry, be happy. In AAMAS 2003, pages 313-320. ACM Press, 2003.

[18] C. Martinho and A. Paiva. Underwater love: Building tristao and isolda's personalities. In M. Wooldridge and M. Veloso, editors, Artificial Intelligence Today. Springer, 1999.

[19] R. Moffat. Personality parameters and programs. In Creating Personality for Synthetic Actors. Springer, 1997.

[20] A. Ortony, G. Clore, and A. Collins. The Cognitive Structure of Emotions. Cambridge University Press, New York, reprinted 1994 edition, 1988.

[21] C. Pelachaud and I. Poggi. Interacting through different modalities: Multimodal communication between synthetic agents. In Proceedings of the working conference on Advanced visual interfaces, 1998. 
[22] R. Plutchik. Evolutionary bases of empathy. In N. Eisenberg and J. Strayer, editors, Empathy and its Development. Cambridge University Press, 1987.

[23] M. Roussos. Issues in the design and evaluation of a virtual reality learning environment (msc). Technical report, University of Illinois at Chicago, 1997.

[24] S. Russell and P. Norvig. Artificial Intelligence: A Modern Approach. Prentice Hall, 1995.

[25] D. Sobral, I. Machado, and A. Paiva. Managing authorship in plot conduction. In G. Goos, H. Hartmanis, and J. Leeuwen, editors, Virtual Storytelling. Springer, 2003.

[26] D. Sobral, I. Machado, and A. Paiva. Managing authorship in plot conduction. In Virtual Storytelling: Using Virtual Reality Technologies for Storytelling. Springer, 2003.

[27] F. Thomas and O. Johnston. The Illusion of Life: Disney Animation. Walt Disney Productions, 1981.

[28] E. Titchener. A textbook of psychology. New York: Mcmillan, 1924.

[29] L. Wispé. History of the concept of empathy. In N. Eisenberg and J. Strayer, editors, Empathy and its Development. Cambridge University Press, 1987.

[30] D. Wolke, S. Woods, H. Schultz, and K. Stanford. Bullying and victimisation of primary school children in soth england and south germany: Prevalence and school factors. British Journal pf Psychology, 92, 2001.

[31] M. Wooldridge. An Introduction to Multi-Agent Systems. John Wiley and Sons, Ltd, 2002. 